\title{
OBACIS: Outcome Based Analytics and Continuous Improvement System
}

\author{
Mohamed A. Ismail, Industrial Systems Engineering, University of Regina, \\ mohamed.ismail@ureinga.ca
}

\begin{abstract}
In this paper, an integrated system for outcome-based assessment and continuous improvement is presented. The system is designed and implemented as a suite of three integrated Apps: An Excel-App for creating Auto Grading Sheets (AGSs); a Web-App for building assessment trees, updating server database(s), uploading associated documents, and conducting surveys; and a Win-App for program-wide and faculty-wide $O B A$ data compilation, performance analysis, and data-informed continuous improvement. The proposed system adopts a bottom-up approach for building assessment trees that define the structure and the smart logic embedded in AGSs. Some course assessment activities, possibly all, are mapped to graduate attributes, more precisely indicators, and course learning outcomes. The proposed system analyzes the collected data from three different views: 1) Categorical Analysis view (CAs), 2) Learning Outcomes Analysis view (LOAs), and 3) Graduate Attributes Analysis (GAAs) view. The paper presents some principles related to the proposed system, demonstrates its multiple user interfaces, and digs more into OBA analytics and its proposed closed-loop continues improvement process. The objective of the proposed system and its underlying framework is to set new grounds for the accreditation process by making it more appealing, more economical, and more fruitful for all involved stakeholders.
\end{abstract}

Keywords: Graduate Attributes, Outcome Based Assessment, Continuous Improvement, Engineering Education

\section{INTRODUCTION}

The Canadian Engineering Accreditation Board (CEAB) outcome-based assessment (OBA) and continuous improvement (CI) process [1] involves many system users, a tremendous amount of data collection, and a treasure of buried information that can dramatically change the way we do our jobs as instructors and the type of engineers our educational facilities can produce in the future. The relatively new process is a radical step forward, but unfortunately, it is still unfathomed, and we are yet to know how we can implement it and create an accompanying closed-loop continuous improvement process. The idea of establishing a new framework that has a clear description of what to be measured and how to take advantage of its outcomes needs further investigation. As reported in [2], The process is still having a significant way to go before its impact is fully realized. Switching from just an event-based process that happens over a course of six years to a continuously improving one makes the process more overwhelming and costly than it simply looks. The last review of the University of Alberta's (UofA) of nine programs required the collection of more than a ton of documents and consumed approximately 16,000 hours in preparation time involving personnel cost of well over $\$ 1 \mathrm{M}$ [3]. For mid-size and small engineering schools, not the size of $U$ of $A$, this could be easily unaffordable and really intimidating. In order to be successful, the process has to be simple, well-defined, and fast. The time of process execution and the time to reap its benefits should be cut down by an order of magnitude.

There is a dearth of software tools reported in the literature for both data collection and program assessment and almost none for continuous improvement purposes. Most of these systems have been designed in an ad-hoc way fulfilling the needs of a specific institution [4]. Since many people are involved in the data collection process, web tools look ideal for facilitating that process: Accreditation Management System (AMS) of U of A [3] and ABET course assessment tool (ACAT) system [5] of the University of Nevada, Reno are typical examples. Some other related tools include a webtool for learning outcomes mapping and a software tool for the administration of the final year engineering projects reported in [4] and [6] respectively. There is still a lack of an integrated system or a suite of tools that addresses all the aspects of outcome-based assessment and its demanding continuous improvement associated process(es).

In this paper, we will present an integrated framework called outcome-based analytics and continuous improvement system (OBACIS) that is designed and developed to accelerate the accreditation preparation process, cut down its costs, integrates grade reporting with OBA reporting, and create intensive set of data analytics that will drive the continuous improvement efforts done at many levels, for many deliverables, and by many stakeholders. The paper is organized as follows: sec 2 introduces some premises that underlie the OBACIS framework design specifications; sec 3 introduces two major system artifacts that help the interested reader better understand the system workings; sec 4 describes the navigation between the three different apps and the process workflow; sec 5 introduces the three reports created by OBACIS and how they can stimulate continuous improvement initiatives; sec 6 reports the current state of development; sec 7 summarizes and concludes the paper. 


\section{OBACIS PREMISES}

The OBACIS project has been developed with the following premises and specifications in mind:

1. Since the CEAB switched to the new OBA \& CI system, the process has changed from being a 6-year event to an ongoing activity with many implications on the teaching and learning workload required by the teaching faculty and higher administration. The data collection adds a new major academic task at the end of every academic semester which could be an inappropriate time to ask professors to do an additional job. If the accreditation process is turned to be an ongoing activity, it should be integrated and streamlined with other grading activities throughout the teaching semester.

2. The new system focuses on mapping the quality of engineering programs and engineering students to a welldefined set of graduate attributes measured by an associated set of indicators [7]. The CEAB OBA \& CI structure is based on Washington Accord, which was mainly based on ABET OBA process developed in the nineties, the rise time of total quality management (TQM). Quality today has a different definition as the inverse of variability that needs to be eliminated by complete devotion to continuous improvement activities - 6sigma practices. Three questions arise here:1) Is the current system of OBA sufficient enough for identifying CI opportunities? 2) Who/what are the targets of the CI process: students, course activities, program curricula, professors' performance, etc.? 3) Should we shift the paradigm from the TQM that is now is fading away everywhere to the more practical and more successful 6-Sigma practices?

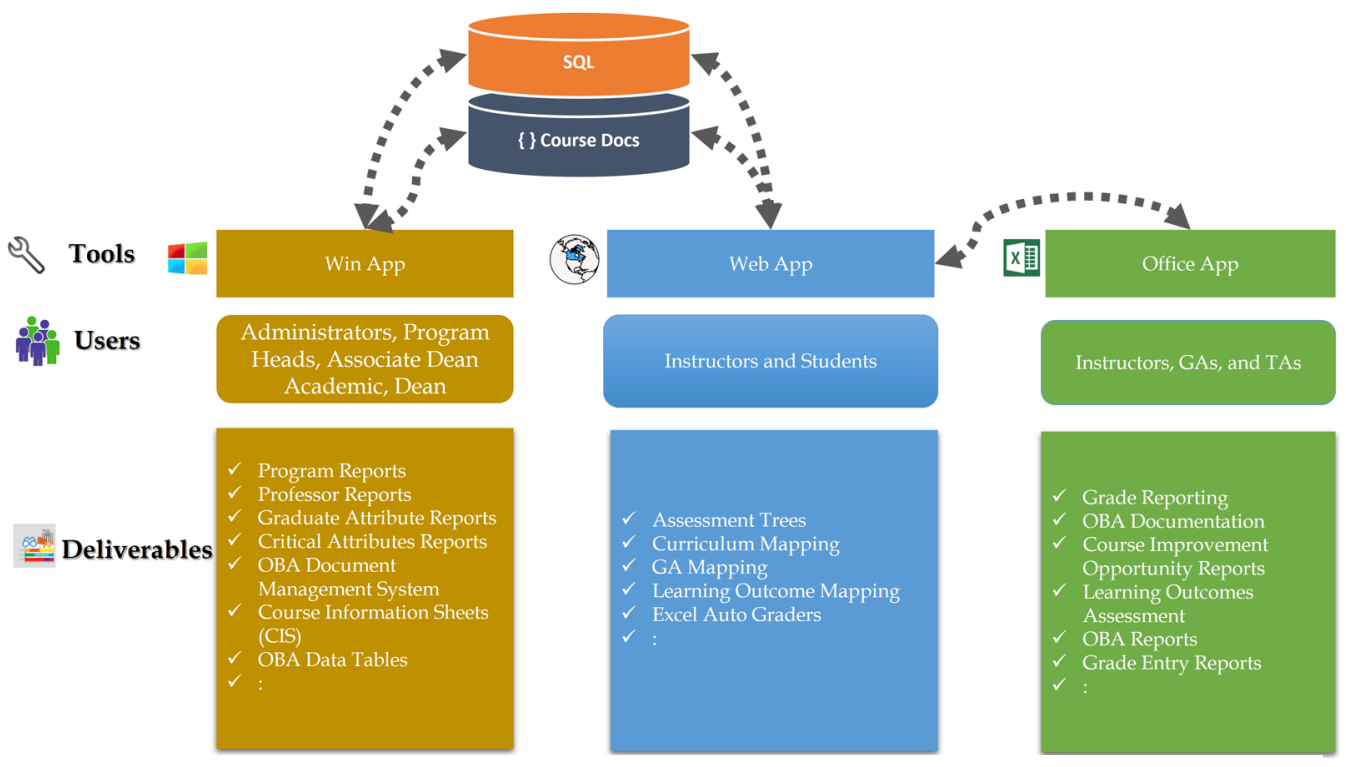

Figure 1: OBACIS Framework

3. Current accreditation reporting uses Excel templates for data collection as the main medium for data collection; a useful template could be downloaded from EGAD website [8]. Despite their ease of use, current templates lack strict structure which might cause a lot of errors during compilation. Using excel for grade reporting and another template for OBA reporting duplicates the process and exhausts both professors and administration. A unified and strictly structured template could be a big time, effort, and money saver.
4. The process should take advantage of today's technologies in data collection, analytics, communication, and closed-loop improvement processes. The system should move the OBA \& CI a step forward by creating the required OBA data and their analyses the moment the grades are recorded. The charts should be very informative and provides enough evidence for continuous improvements action plans.

All of these premises have been developed over time while working on the OBACIS development. The first version was just an Excel Add-in developed to automate OBA reports creation. The first generation introduced the main three reports of categorical analysis (CA), learning outcomes analysis (LOA), and outcome-based analysis (OBA). The second generation was to extend the framework and make it useable at both program and faculty levels. After many iterations, the latest system architecture has been developed as shown in Figure 1. The developed system is composed of three different pieces of software forming an integrated suite: An Excel APP, a Web-App, and a Desktop or a Win-App. The Excel App is used for creating Auto Grading Sheets (AGSs), cf. sec 3.1 and sec 3.2; The Web-App is used for building assessment trees, updating server database(s), uploading associated documents, and conducting surveys; and the Win-App for program-wide 


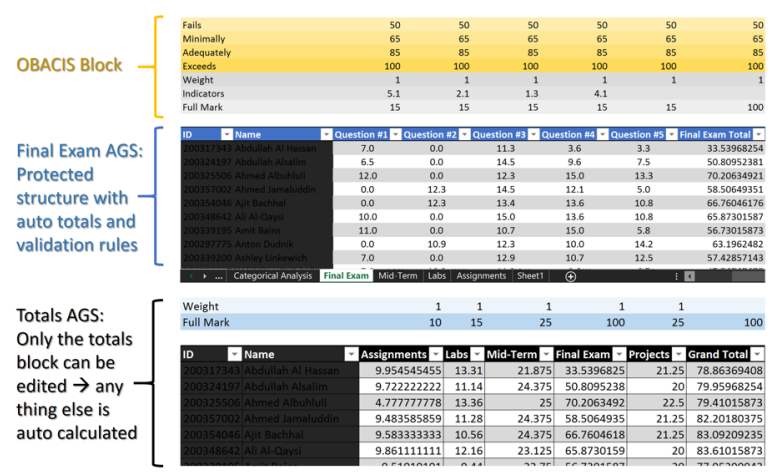

Figure 2: OBACIS Auto Graded Sheet (AGS)

aggregation-evaluating a total course grade or assigning a grade letter. Students data are obtained from the registrar's database via administration office or a web-service. Once, the semester is over, all students' data are compiled, aggregated, and then reported to the administration, uploaded to a webservice, or updated using a web-app connected to university's registrar's student database. Those excel sheets are created on an ad-hoc basis, and there are no guidelines to create them or archive them. The process is time-consuming, and a lot of care is required to make the process error free. This routine work is done by almost every instructor for nearly every course being taught. The CEAB OBA \& CI process requires further processing of those grading sheets according to a predefined curriculum map developed by program faculty to relate assessment activities to graduate attributes via associated set of indicators measured by a set of performance descriptors [7]. An Excel workbook based on special OBA rubric is required, uploaded, and later compiled by the administration for accreditation reporting purposes. The first artifact that our framework introduces is the Excel Auto Grading Sheets (AGSs), see Figure 2. An AGS is a unified grading worksheet that helps in grade reporting, accreditation reporting, and creating the three most important reports generated by our system, CA,LOA,OBA reports. AGSs are structured to match course assessment activities reported in the course syllabus and are classified by their different categories: Assignments, Labs, Mid-Term, etc. An OBACIS Block is introduced on the top every worksheet. This block determines performance descriptors for accreditation reporting purposes [7]. All grades must be recorded on an individual basis (question by question or report by report, or lab by lab and so forth). This complies with CEAB questionnaire description of assessment tools bookkeeping [7]. The remaining parts of the block represent grade weights (could be any non-zero figures and don't need to add up to 1 or $100 \%$ ), indicators list (must be consistent with the program curriculum map), and full mark for each grade item and the entire category. The AGSs are created, formatted, and has a few limited regions/cells that can be edited by an AGS user, a strict a structure and layout requirement. The structure of those sheets and the OBABCIS block is created using Web-app and an Excel app loaded to the server after an interactive session between the Web-app user, cf. sec 3 and sec 4 . The creation of those AGSs is the heart of the OBACIS workflow and they are auto-created after defining course assessment trees, cf. sec 3.2.

\subsection{Assessment Trees}

Assessment trees are instrumental in creating AGSs, OBACIS block, and OBACIS reports. Course assessment tools or grade items could be organized as a hierarchy of

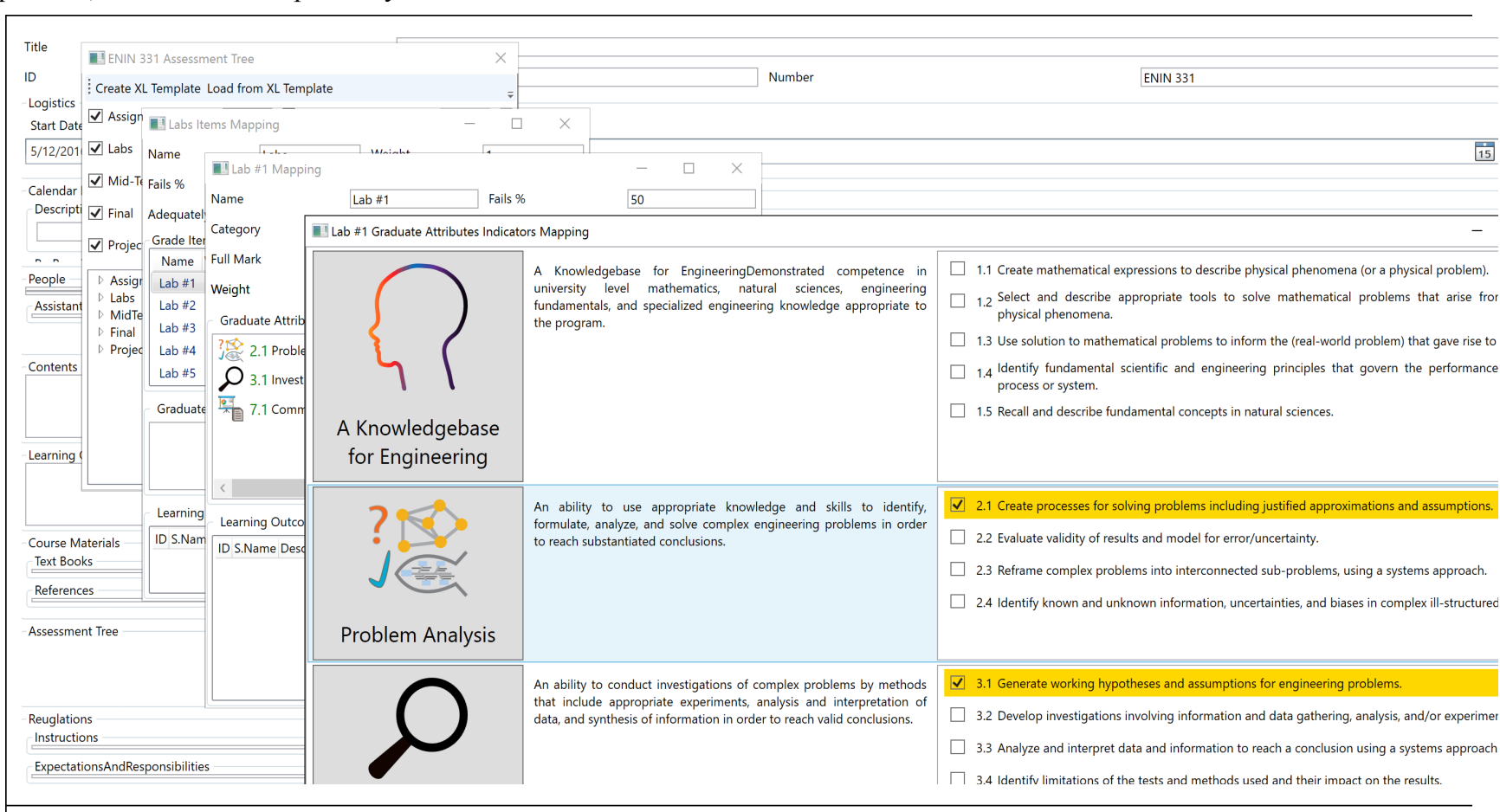

Figure 3: Assessment tree building via Win-App

CEEA16; Paper 141

Dalhousie University; June 19 - 22, 2016 
categories, sub-categories, and individual grade items. This hierarchy is represented as a tree with its root is the course name itself. Grade items are the leaves of the tree while categories and sub-categories are the internal nodes. Every leaf represents a grade item which can be assigned a full mark, weight, and performance descriptors, a set of learning outcomes and a set of graduate indicators. Internal nodes have two independent sets for learning outcomes and indicators sets. Those sets are seamlessly updated as an aggregation of those defined for end nodes or grade items. Assessment trees are easily built using the web-app or the win-app by utilizing either the user-friendly web forms or win forms. Figure 3 shows the creation process of a course assessment tree. Assessment trees and registered students' data (Names and IDs) are the main constituents of AGSs. Both combined define the read-only and editable ranges of those sheets. The editable ranges allow copying and pasting, which make the professor/instructor job much easier by creating multiple copies for TAs and Lab instructors to fill them up independently for later compilation.

\section{PROCESS WORKFLOW}

Both the accreditation and the grade reporting processes start simultaneously by logging on to the web-app page and requesting a new course AGSs template, see Figure 4. The course ID is required to access students' database and update the list of registered students' names and IDs, see Figure 4. Through a set of interactive web forms, similar to their win- app counterparts shown in Figure 3, the system interacts with the user to collect data required for OBACIS block followed by mapping both the learning outcomes and graduate attribute indicators to the appropriate grade items. Once the data entry process is complete, the AGSs are created using the Excel App on the server and should be easily downloaded for grade updating. AGSs are a strictly structured workbook populated with OBACIS blocks, grading sheets, auto totals columns, validation rules, and a set of templates for CA, LOA, OBA reports. The moment a professor enters all course grades; the totals are immediately calculated, and all OBACIS reports are updated. Professors are now more capable of evaluating their teaching experiences and pondering about course deliverables improvements using CA reports, learning outcomes improvements using LOA reports, and graduate attribute improvements using OBA reports, cf. sec 5. From here, signals for adaptations will be captured, improved action plans could be jotted down, and the closed-loop starts to take shape.

Once the grades have been approved by the higher administration, the AGSs are ready for uploading using the Web-app for program-wide and faculty-wide data compilation and warehousing. The web app can save the AGSs file into faculty-wide OBA repository. The web app can transform AGSs into an XML to be saved to system docs or repository or to a relational database such as SQL DB, for archiving and future use, see Figure 4. Year to year performance improvements, 3 years' improvements, or 5 years' improvements reports could be at the disposal of top administration and OBA committees to drive the quality

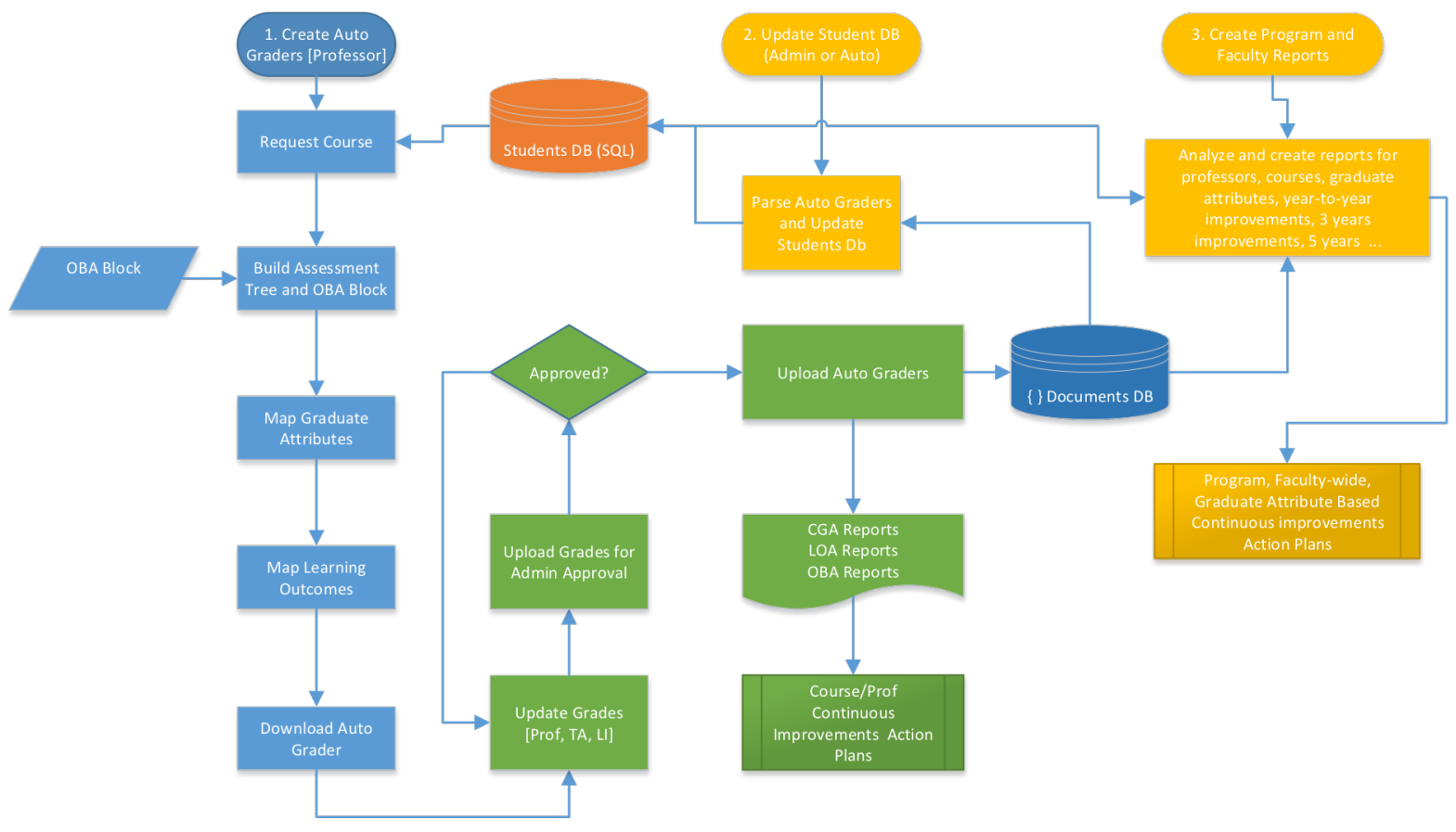

Figure 2: OBACIS workflow chart. Blue elements represent the web-app related activities; the green ones represent the grade reporting process using AGSs; and the golden ones represent the Win-app activities. System Databases(s) and Document Base represent the bridges between the three systems. 
administration of the teaching and learning process and its stakeholders. These reports are created by the last but not the least piece of the puzzle - the Win app.

Win-app is only accessible by top administration and system administers. Performance monitoring and backtracking reports for students, faculty, courses, and even TAs/GAs can now be easily generated. In addition, the app is capable of generating OBA course information sheets (CIS), data tables, and most of the pieces of information required by the latest CEAB accreditation questionnaire [7]. All sorts of quantitative and statistical analyses can be created to assess the teaching and learning quality and sets the grounds for a grand ' $6-\sigma$ like' educational project.

\section{OBACIS ANALYTICS AND REPORTS}

The CEAB mandates using performance descriptors to assess graduate attributes by measuring students' achieved levels of mapped indicators. Figure 5a shows OBACIS stepped bar chart that depicts those descriptors. Four descriptors have been chosen identical to those recommended by EGAD project Excel template[8]. OBACIS allows the user to change those descriptors any time during data entry using web-app or winapp or later during grade recording process using AGSs.

In order to get closer to the 6-sigma quality best practices, we started to look for a way to measuring variability in a teaching and learning setup. The solution was a normalized box plot chart shown in Figure 5b. All grade items are calculated as percentages; a comparative box plot was developed. Normalized box plots are ideal for creating comparative studies, and they were instrumental in creating the three reporting views of OBACIS analytics: CA, LOA, OBA. Continues improvement efforts should lead to box plot leveling where there should be an almost imaginary horizontal straight line that connects the medians of all course activities. Moving upward from such an imaginary line should reflect continuous improvement efforts from one semester to another. Using these box plots, we can compare different grade items for different indicators, different learning outcomes, different courses, different professors and different programs.

FINAL EXAM

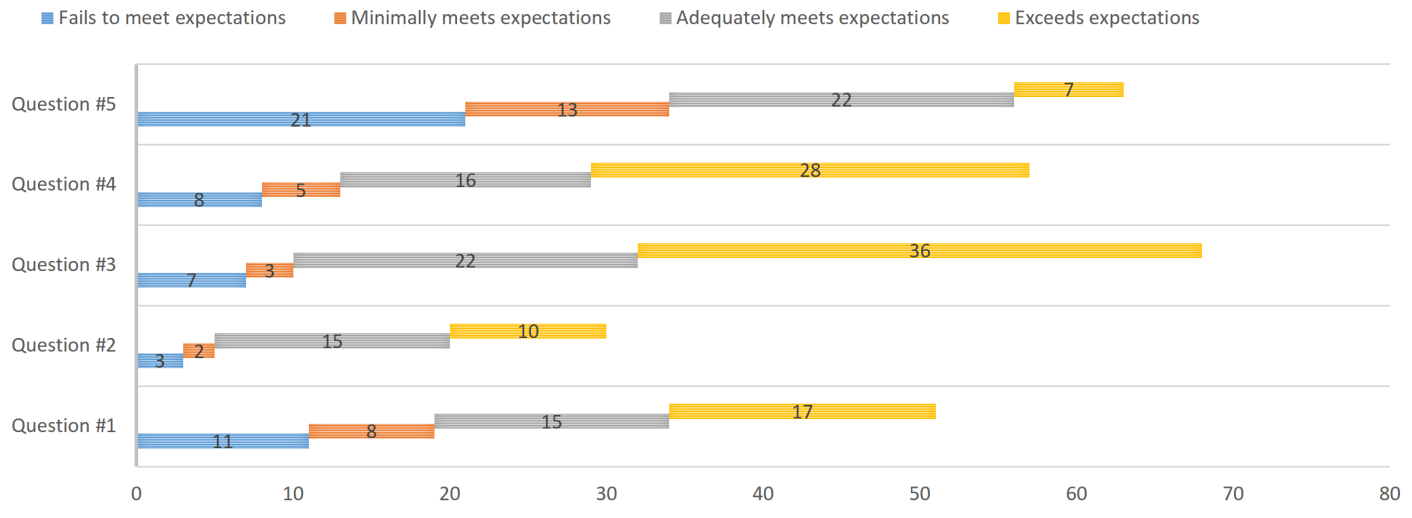

Figure 5a: Categorical Analysis, Final Exam Stepped Bar Chart

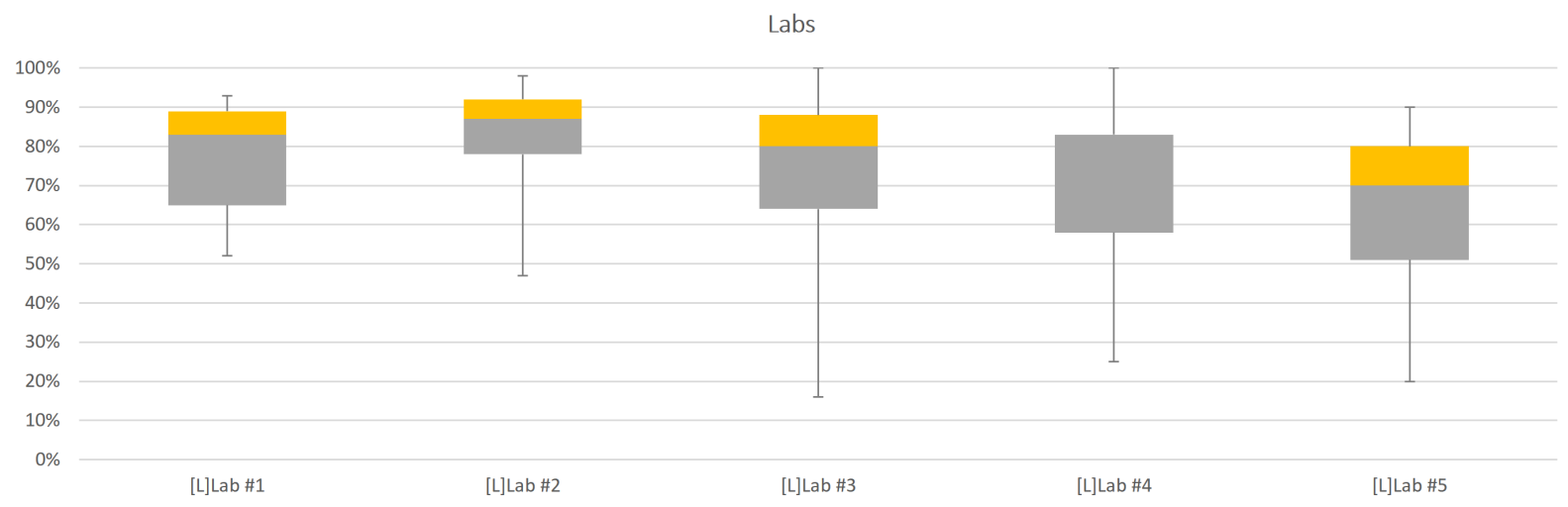

Figure 5b: Categorical Analysis: Labs Performance Normalized Box Plot. Lab \#5 is red flagged, lowest average highest variability

CEEA16; Paper 141

Dalhousie University; June 19 - 22, 2016 


\subsection{Categorical Analysis (CA)}

All the assessment elements conducted throughout the course are analyzed statistically in order to identify the gaps and weaknesses that should be addressed in the following teaching semester(s). The focus of this type of analytics is on course components or categories. Figure 6 shows the results of an industrial engineering class taught in Fall 2014. By just having a quick look to categorical charts, we can see in which component we are falling behind and what needs to be addressed during the next round of teaching. Assignable causes now can be identified, and action plans could be executed either to fix a certain issue or to improve the delivering method of a certain piece of course content. contents/labs/assignments to see what are the sources of low performance in terms of average or high levels of variability. $\mathrm{CA}$ is our proposed tool for data-driven course design and methods of delivery continuous improvement initiatives.

\subsection{Learning Outcomes Analysis (LOA)}

In this part, grade items are mapped to course learning outcomes (LOs). LOs list is a required part of course information sheets (CIS) that need to be reported for all courses for accreditation purposes [7]. The LOs assessment and their involvement in the continuous improvement activities are optionally offered by OBACIS framework and not required by $\mathrm{CEAB}$ accreditation. Any number of grade items or course activities could be mapped to learning

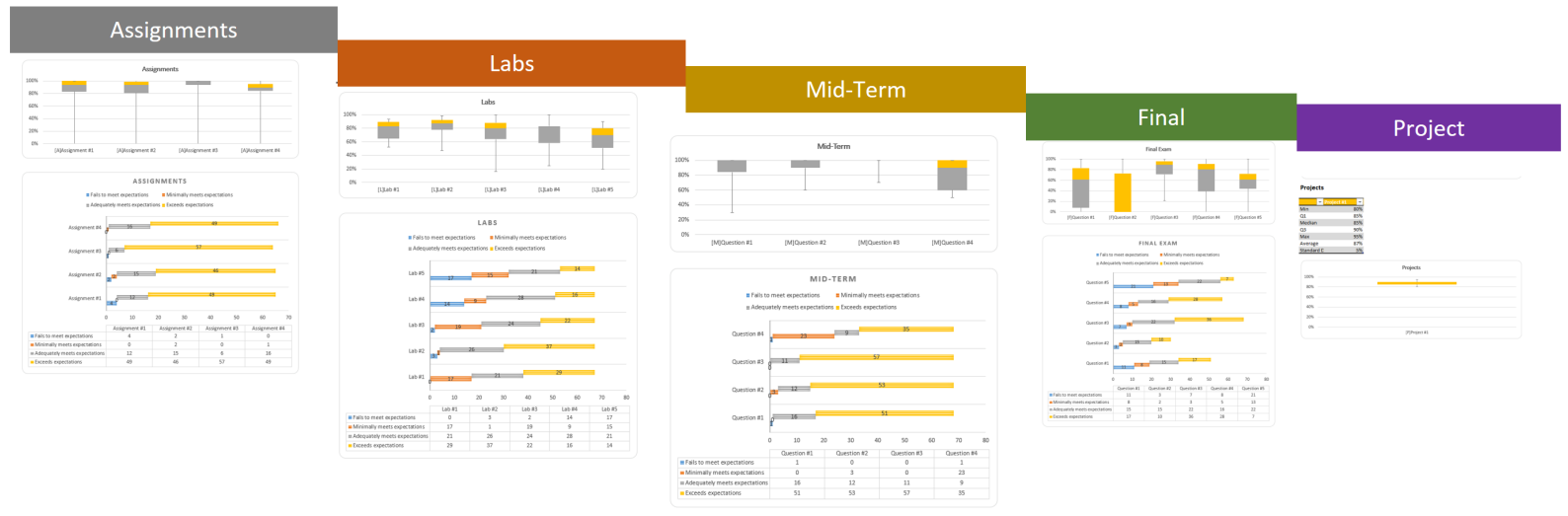

Figure 6: Categorical Analysis: Which category(ies)? Labs and Final; Labs 4 \& 5 needs further investigation for assignable causes; Final Q1? Q2? Q5; Project 1 box plot? A rubric is desperately needed

By analyzing Figure 6, we can find that Labs and the Final Exam are the most revealing categories. Labs 4 and 5 need further investigation. A constructive discussion with the course lab instructor can lead to some modifications of lab material, more lab time assignment for those activities, better demonstrations, etc. Should I as a professor work more on those late lectures? By analyzing the final charts, a reverse mapping now is required for Q1, Q2, and Q5 to course outcomes, see sections 3 and 4 for details. Similar to CA, box plots and stepped bar charts are created by OBACES. By reviewing those charts, we can pinpoint immediately which learning outcome that doesn't have enough assessment tools or which learning outcomes that need immediate attention, see Figure 7. All learning outcomes must be achieved over time.
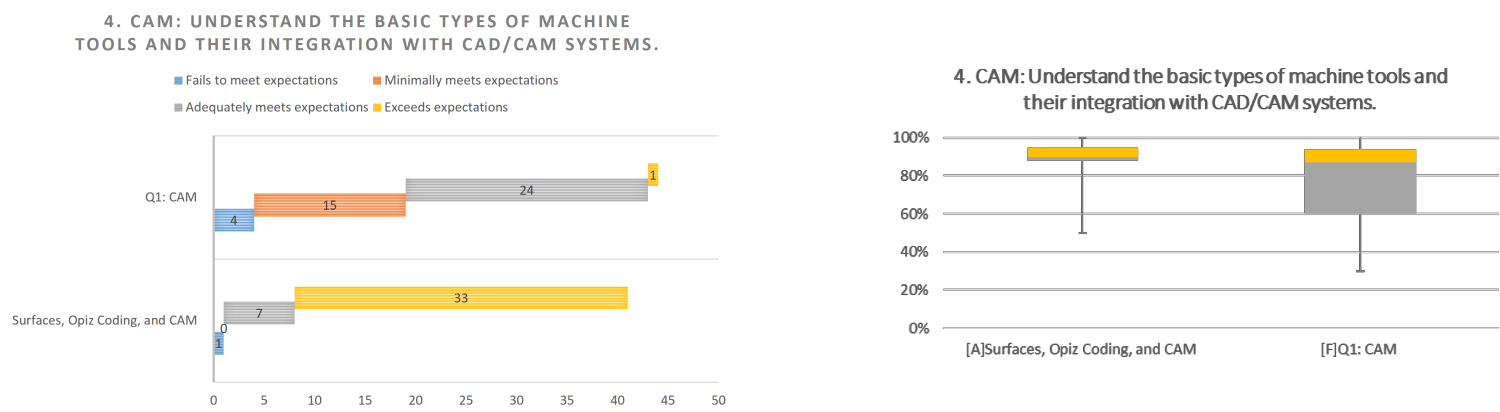

Figure 7: CAD/CAM course Learning outcome \#4 analysis. Assessment tools: 1 assignment and 1 question in the final. $25 \%$ got less than $60 \%$ in that question, the second $25 \%$ between $60 \%$ and $84 \%$. The variability of Q1 is higher than that of assignment, which is a typical expectation. Assignments should be recorded on a question by question basis; unfortunately that was not the case during that semester 


\subsection{Outcome Based Assessment Analysis (OBA)}

This the closest part of OBACIS analytics that complies with the accreditation process requirements by CEAB. In this part, some grade items or assessment tools are mapped to OBA indicators and analyzed from that perspective. The power of the 12 graduate attributes assessment system is that they are universal and can be applicable to any number of grade items. By using comparative box plots, we can use mapped indicators to evaluate one course, multiple courses, or an entire curriculum. The system can also be used for students', professors, and programs performance evaluation and comparative studies. The only concern of the OBA analytics, from our perspective, is that they are not close enough to the low levels of teaching and learning process, especially the classroom delivery and course design activities. We believe that CAs and LOA could drive the CI efforts better at those lower levels. The best part is that OBA indicators improvements are tightly correlated to the actions taken based on CA and LOA analyses. With OBACIS, we can assign as many indicators as we wish for any grade item or assessment tools. See Figure 8. The question that arises is which grade item performance we should consider for higher levels of reporting? The most critical attribute, see figure 8 , should be $\mathrm{Q} 2$. Since the win-app can digest massive amounts of data, the answer can be delayed until administration determines which attribute is the most appropriate. Graduate attributes should comply with the curriculum map and should stay the same at least for a certain number of teaching cycles or rolling horizons.

Based on personal experience, experiential learning activities and assessment tools are of a paramount importance for the success of the new graduate attributes system. Activities like course projects, term papers, case studies, capstone projects, and essays are the most matching activities with the graduate attributes matrix and cover a wide range of those indicators. Those activities could be simply classified as complex problems solving activities as defined in [7]. More interest needs to be made to those activities, more course grades should be assigned for them, more time should be allocated, and the faculty who inject those activities into program curricula must be rewarded. Rubrics rather than classical grading schemes are more suitable for those types of activities. The top administration should encourage those activities and makes rubric development a common engineering teaching practice.

After compiling and persisting students grading data using the web-app, the win-app, maybe any time later, can generate a student by student graduate attributes development reports.

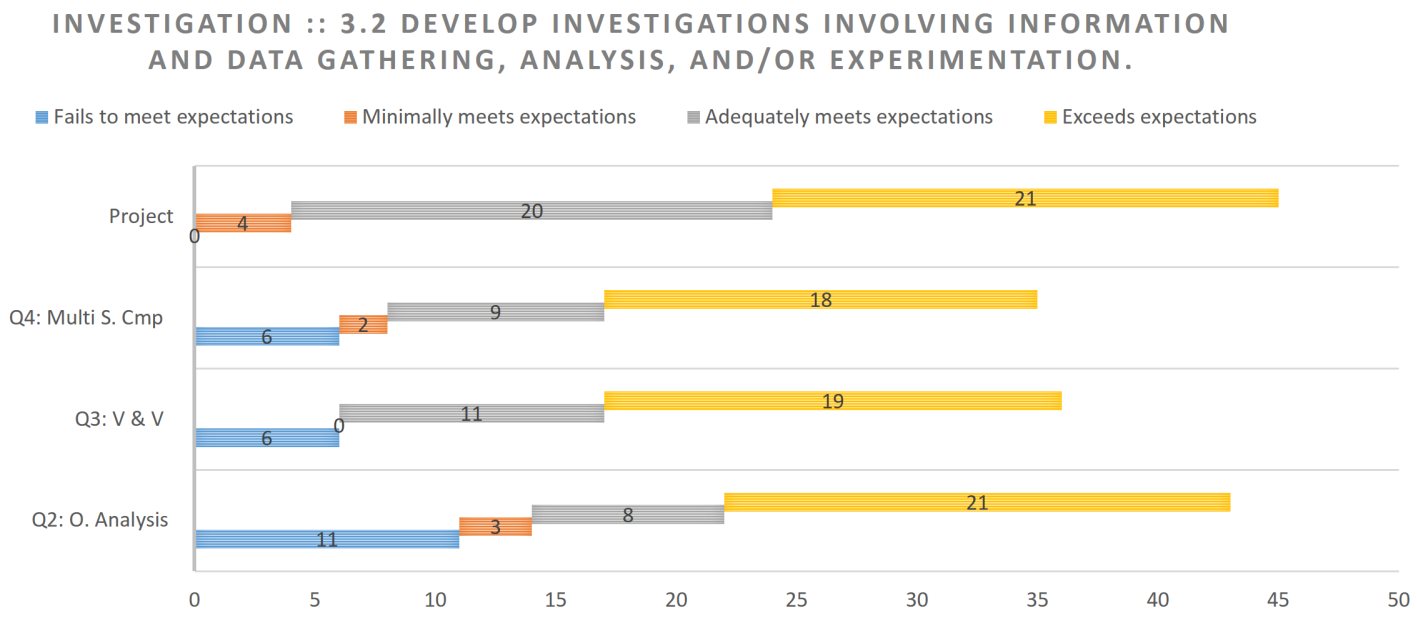

Investigation :: 3.2 Develop investigations involving information and data gathering, analysis, and/or experimentation.

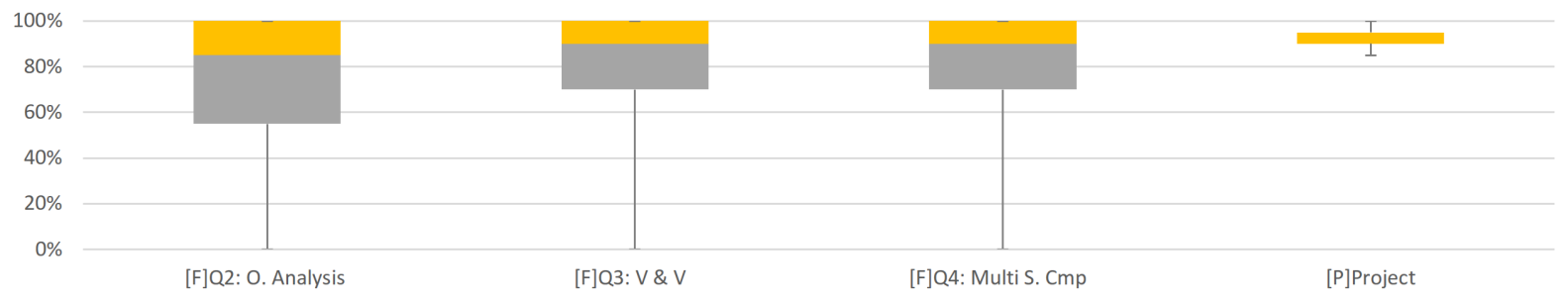

Figure 8: Indictor 3.2 mapped to 4 different course grade items; which item we should report? 
Student historical records can be tracked down and reported to students on an annual or a semester basis over their course of engineering studies. Such reports will engage the students in the new process and will let them pay attention for their graduate attributes achievement records which should prepare them for a more successful engineering career. By analyzing such reports, mentoring activities can be offered by engineering faculty or a dedicated faculty member in every program. Certain skills development workshops could be recommended and offered to catch up or to gain some critical attributes that our curricula might fail to develop. By aggregating data or sampling data, program performance reports can be generated the same way. By identifying critical attributes at the program level, program curriculum could be revised and developed to improve the performance of those attributes.

\section{SUMMARY AND CONCLUSIONS}

OBACIS framework has been developed to facilitate the collection, organization, analysis and presentation of the data required to demonstrate compliance with $\mathrm{CEAB}$ requirements. The system streamlines the documentation process and serves several stakeholders from professors to program heads, and both faculty and university top administration. The system is versatile and expands on the accreditation requirements to create a comprehensive, more appealing, and less costly process in terms of both time and money. OBACIS introduces an integrated closed-loop system(s) that may act as a proactive driver for creating multidimensional continuous improvement processes that should enhance the performance of our educational institutions and all the involved stakeholders. The system measure and analyze output data from three different perspectives: Categorical Prospective, Learning Outcomes Perspective, and Outcome Based Perspective. A suite of three apps that implement the proposed system and streamline both grade and accreditation reporting processes has been presented. The suite includes an Excel APP responsible for creating Auto Grading Sheets, a web app for data transfer, compilation, and reporting, and a win-app for centralized data processing, analytics, and higher levels reporting used by top administration to drive the entire reporting and continuous improvement activities. The proposed system is an initiative to close the continuous improvement loop and getting a bit closer from today's prevalent 6-sigma practices.

\section{REFERENCES}

1. 2015 Accreditation Criteria and Procedures in Canadian Engineering Accreditation Board (CEAB).

2. Spracklin-Reid, D. and A. Fisher, Course-Based Learning Outcomes as the Foundation for Assessment of Graduate Attributes, in Canadian Engineering Education Association (CEEA12)2012: Winnipeg, MB.
3. Dew, S.K., M. Lavoie, and A. Snelgrove, An engineering accreditation management system, in Canadian Engineering Education Association 2nd Conference 2011, June: St. John's, Newfoundland, Canada.

4. Ibrahim, W., et al., A Web-Based Course Assessment Tool with Direct Mapping to Student Outcomes. Educational Technology \& Society, , 2015. 18 (2): p. 46-59.

5. Essa, E., et al., ACAT: a web-based software tool to facilitate course assessment for ABET accreditation, in The 7th International Conference Information Technology2010: Las Vegas, USA.

6. Grobler, M.J., H.-J. Marais, and M. Delport, $A$ software tool for the administration of final year engineering projects, in AFRICON2013: PointeAux-Piments.

7. CEAB, Questionnaire for Evaluation of an Engineering Program - Exhibit 1, 2016.

8. http://engineering.queensu.ca/egad. Engineering Graduate Attribute Development (EGAD) Project Last Accessed May, 2016 